\title{
DYNAMICS OF A QUASI-QUADRATIC MAP
}

\author{
ASSIS AZEVEDO, MARIA CARVALHO, AND ANTÓNIO MACHIAVELO
}

\begin{abstract}
We consider the map $\chi: \mathbb{Q} \rightarrow \mathbb{Q}$ given by $\chi(x)=x\lceil x\rceil$, where $\lceil x\rceil$ denotes the smallest integer greater than or equal to $x$, and study the problem of finding, for each rational, the smallest number of iterations of $\chi$ that eventually sends it into an integer. Given two natural numbers $M$ and $n$, we prove that the set of irreducible fractions with denominator $M$ whose orbits by $\chi$ reach an integer in exactly $n$ iterations is a disjoint union of congruence classes modulo $M^{n}$, establishing along the way a finite procedure to ascertain them. We also describe an efficient algorithm to decide if an orbit fails to hit an integer until a prescribed number of iterations, and deduce that the probability that an orbit enters $\mathbb{Z}$ is equal to one.
\end{abstract}

\section{INTRODUCTION}

Let $\chi: \mathbb{Q} \rightarrow \mathbb{Q}$ be the map given by $\chi(x)=x\lceil x\rceil$, where $\lceil x\rceil$ denotes the smallest integer greater than or equal to $x$, and consider the orbits $\left(\chi^{n}(x)\right)_{n \in \mathbb{N}_{0}}$ of any $x \in \mathbb{Q}$. We note that $\mathbb{Z}$ is invariant by $\chi$, the fixed points are the rational elements in $\left.\left.[0,1], \chi^{-1}(\{0\})=\right]-1,0\right], \chi(-x)=\chi(x)-x$ if $x \in \mathbb{Q} \backslash \mathbb{Z}$, and that, if $x \leq-1$, then $\chi(x) \geq 1$.

For $\frac{p}{q} \in \mathbb{Q} \cap\left[1,+\infty\left[\right.\right.$, where $p, q$ belong to $\mathbb{N}$ and $(p, q)=1$, the iterate $\chi^{j}\left(\frac{p}{q}\right)$ is an irreducible quotient $\frac{p_{j}}{q_{j}}$, where $q_{j+1}$ divides $q_{j}$. Therefore the sequence of denominators $q_{j}$ is decreasing, although not strictly in general. For instance, the first iterates of $\frac{31}{10}$ are

$$
\frac{62}{5}, \quad \frac{806}{5}, \quad \frac{130572}{5}, \quad 681977556
$$

The number of iterates of $\frac{p}{q}$ needed to hit an integer may be as large as we want. In fact, if we have any finite sequence of positive integers $\left(q_{j}\right)_{j \leq n}$ where $q_{i+1}$ divides $q_{j}$ for any $j$ then there exists $x \in \mathbb{Q}$ such that $\chi^{j}(x)=\frac{p_{j}}{q_{j}}$ with $\left(p_{j}, q_{j}\right)=1$ (see Remark 2.1). However, numerical evidence suggests that, for any such $\frac{p}{q}$, there is a $j \in \mathbb{N}$ verifying $q_{j}=1$. This behaviour bears a resemblance to the dynamics of $G: \mathbb{Q} \cap[0,1] \rightarrow \mathbb{Q} \cap[0,1], G(x)=\frac{1}{x}-\left[\frac{1}{x}\right], G(0)=0$, although in this case the orbit of each rational number in $[0,1]$ is a sequence of irreducible fractions whose denominators decrease strictly before it ends at 0 , and this happens in finite time.

For $x \in \mathbb{Q}$, define the order of $x$ as

$$
\operatorname{ord}(x)=\min \left\{k \in \mathbb{N}_{0}: \chi^{k}(x) \in \mathbb{Z}\right\}
$$

Date: 29 September 2012.

2010 Mathematics Subject Classification. 11A07, 37P99.

Key words and phrases. Discrete dynamical system, ceiling function, density, covering system.

This work was partially supported by Fundação para a Ciência e Tecnologia (FCT), through Centro de Matemática da Universidade do Minho and Centro de Matemática da Universidade do Porto, FCT project UT-Austin/MAT/0035/2008 and Program POSI. 
if this set is nonempty, and $\operatorname{ord}(x)=\infty$ otherwise. The integers are the elements of order 0 ; the rational numbers in $] 0,1[$ have infinite order. It is easy to evaluate the order of any irreducible fraction $\frac{a}{2}$ in $\mathbb{Q} \cap[1,+\infty$ [: given an odd $a \in \mathbb{N}$, say $a=2^{k} b+1$ for a positive integer $k$ and an odd $b$, using induction on $k \in \mathbb{N}$ and the equality

$$
\chi\left(\frac{a}{2}\right)=\frac{2^{k-1} b\left(2^{k} b+3\right)+1}{2},
$$

one has ord $\left(\frac{a}{2}\right)=k$. In particular, for each $k \in \mathbb{N}$, the smallest positive irreducible fraction with denominator 2 whose order is $k$ is $\frac{2^{k}+1}{2}$. We note that not only this smallest value increases with $k$, but it does so exponentially. On the other hand, the following table, which displays the smallest integer $a$ such that $1 \leq \operatorname{ord}\left(\frac{a}{3}\right) \leq 50$, shows that, within the rational numbers with denominator 3 , that no longer holds.

\begin{tabular}{|r|r||r|r||r|r||}
\hline order & smallest integer $a$ & order & smallest integer $a$ & order & smallest integer $a$ \\
\hline \hline 1 & 7 & 18 & 2215 & 35 & 6335903 \\
2 & 4 & 19 & 6151 & 36 & 1180939 \\
3 & 13 & 20 & 8653 & 37 & 1751431 \\
4 & 20 & 21 & 280 & 38 & 10970993 \\
5 & 10 & 22 & 28 & 39 & 17545207 \\
6 & 5 & 23 & 1783 & 40 & 66269497 \\
7 & 29 & 24 & 81653 & 41 & 27952480 \\
8 & 76 & 25 & 19310 & 42 & 60284614 \\
9 & 50 & 26 & 114698 & 43 & 203071951 \\
10 & 452 & 27 & 18716 & 44 & 191482466 \\
11 & 244 & 28 & 196832 & 45 & 144756173 \\
12 & 830 & 29 & 15214 & 46 & 45781445 \\
13 & 49 & 30 & 7148 & 47 & 1343664136 \\
14 & 91 & 31 & 273223 & 48 & 223084774 \\
15 & 319 & 32 & 3399188 & 49 & 1494753473 \\
16 & 2639 & 33 & 398314 & 50 & 20110862 \\
17 & 5753 & 34 & 6553568 & & \\
\hline
\end{tabular}

FIGURE 1. Smallest positive integer $a$ such that $\frac{a}{3}$ has order between 1 and 50 .

We have also verified that, for $a \leq 2000000000$, the order of $\frac{a}{3}$ is equal or less than 56. Clearly, this computation was not achieved directly from the definition of order, because the iterates grow very rapidly: for example, $\frac{28}{3}$ has order 22 and $\chi^{22}\left(\frac{28}{3}\right)$ is an integer with 4134726 digits. Our numerical experiments were possible due to two redeeming features: the dynamical nature of the problem, which allowed us to reduce the difficulty in each iteration; and, moreover, the location of the rational numbers with given denominator and a fixed order among the elements of specific congruence classes modulo a certain power of the denominator, which enabled us to deal only with numerators that are limited by that power.

In what follows, and after showing that the elements of order $n$ lay in some congruence classes, we give in Theorem 2.3 a recursive formula for the number of those classes. We use it to attest that the natural, or asymptotic, density (see [3], p. 270) of the elements of $\mathbb{Q} \cap[1,+\infty[$ that have infinite order is zero (this 
follows from Theorem 3.1). We then present an efficient algorithm to determine if a rational number has an order below a given bound. Finally we comment on some alternative approaches and affinities that this problem seems to have with the Collatz conjecture and the Erdös-Straus conjecture on unit fractions.

\section{NuMBERS OF ORDER $n$}

We are not aware of any efficient algorithm to evaluate the order of a rational number. We also do not know if there are numbers, besides the ones in the interval ] 0,1 [, with infinite order. But we do have two algorithms to decide if a rational $\frac{a}{M}$ has order $n$, for a fixed $n$. We will see that, in both cases, one only needs to consider $a<M^{n+1}$.

The first algorithm gives a way to find all the elements of order $n$ if one knows all the elements of order $n-1$, and it is a subproduct of the results in this section. It relies on the resolution of a number of quadratic congruences that increases exponentially with $n$, but what is more significative is that one gets substantial information on the structure of the elements of order $n$, which is sufficient to prove that almost all elements have finite order. The second algorithm will be presented in section 4 .

The underlying basic idea is simply to use the obvious fact that ord $(\chi(x))=$ $\operatorname{ord}(x)-1$, for each $x \in \mathbb{Q} \backslash \mathbb{Z}$, to find information about the elements of order $n$ from the ones of order $n-1$, somehow reversing the dynamics of the map $\chi$. Surely, given $y \in \mathbb{Q}$, there is not in general a rational $x$ such that $\chi(x)=y$ (consider $y=\frac{5}{3}$, for example). But, in the proof of Theorem 2.3. we prove that, in some sense, the process is reversible.

Let us start by caracterizing the elements that have order equal to 1 .

Lemma 2.1. Let $x=\frac{a}{M}$, where $a \in \mathbb{Z}, M \in \mathbb{N}$, with $M>1$, and $(a, M)=1$. Then $\operatorname{ord}(x)=1$ if and only if there exists $r \in\{1,2, \ldots, M\}$ such that $(r, M)=1$ and $a \equiv-r\left(\bmod M^{2}\right)$.

Proof. We note first that $x \notin \mathbb{Z}$, and also that $x$ has order 1 if and only if $\chi(x) \in$ $\mathbb{Z}$, which is equivalent, since $(a, M)=1$, to the condition that $M$ divides $\left\lceil\frac{a}{M}\right\rceil$. Consider $k \in \mathbb{Z}$ and $0<r<M^{2}$ such that $a=k M^{2}-r$; notice that, as $(a, M)=1$, we have $(r, M)=1$ and $r \neq 0$. Then $\left\lceil\frac{a}{M}\right\rceil=k M+\left\lceil\frac{-r}{M}\right\rceil$ and so

$$
\begin{aligned}
M \text { divides }\left\lceil\frac{a}{M}\right\rceil & \Leftrightarrow M \text { divides }\left\lceil-\frac{r}{M}\right\rceil \\
& \Leftrightarrow\left\lceil-\frac{r}{M}\right\rceil=0, \text { as }-M<-\frac{r}{M}<0 \\
& \Leftrightarrow r \in\{1,2, \ldots, M-1\} .
\end{aligned}
$$

This lemma provides the basis for the induction in the proof of the following result.

Proposition 2.2. If $n \in \mathbb{N}$, then, for all $M \in \mathbb{N}$, the set

$$
\mathcal{A}_{n, M}=\left\{a \in \mathbb{Z}:(a, M)=1, \text { ord }\left(\frac{a}{M}\right)=n\right\}
$$

is a disjoint union of congruence classes modulo $M^{n+1}$.

Proof. If $n=1$, the result is given by the previous Lemma. When $n>1$, we only need to guarantee that, if $a \in \mathbb{Z},(a, M)=1$ and ord $\left(\frac{a}{M}\right)=n$, then 
ord $\left(\frac{a}{M}+t M^{n}\right)=n$, for all $t \in \mathbb{Z}$. Now, if $\chi\left(\frac{a}{M}\right)=\frac{a^{\prime}}{M^{\prime}}$, where $a^{\prime} \in \mathbb{Z}$ is such that $\left(a^{\prime}, M^{\prime}\right)=1$, and $M^{\prime}$ is a divisor of $M$, then

$$
\begin{aligned}
\chi\left(\frac{a}{M}+t M^{n}\right) & =\left(\frac{a}{M}+t M^{n}\right)\left\lceil\frac{a}{M}+t M^{n}\right\rceil \\
& =\left(\frac{a}{M}+t M^{n}\right)\left(\left[\frac{a}{M}\right]+t M^{n}\right) \\
& =\chi\left(\frac{a}{M}\right)+m M^{n-1}, \text { for some } m \in \mathbb{Z} \\
& =\frac{a^{\prime}}{M^{\prime}}+m M^{n-1} .
\end{aligned}
$$

Noting that ord $\left(\frac{a^{\prime}}{M^{\prime}}\right)=n-1$, the result follows by induction on $n$.

From this Proposition we conclude that, when looking for rational numbers with order $n$ in $\mathcal{A}_{n, M}$, we need only to deal with irreducible fractions $\frac{a}{M}$ verifying $a \in$ $\left\{0,1, \ldots, M^{n+1}-1\right\}$. Thus it is now easy to give examples of elements with order $n$. For instance, it is straightforward to conclude by induction on $n$ that, if $p$ is an odd prime number and $n \in \mathbb{N}_{0}$, then the following numbers have order $n$ :

$$
\frac{(p-1) p^{n}+1}{p} ; \frac{(-1)^{n} p^{n}+p-1}{p} ; \frac{-(n+1) p^{n}+p^{n-1}+1}{p}\left(\forall_{n \geq 2}\right) \text {. }
$$

Denote by $A(n, M)$ the number of congruence classes modulo $M^{n+1}$ in $\mathcal{A}_{n, M}$ and by $\varphi$ the Euler function. We have already seen that, for all $n \in \mathbb{N}$,

$$
\left\{\begin{array}{l}
A(0,1)=1, \\
A(n, 1)=0,
\end{array} \text { and, for } M>1, \quad\left\{\begin{array}{l}
A(0, M)=0 \\
A(1, M)=\varphi(M) \text { (by Lemma 2.1). }
\end{array}\right.\right.
$$

It turns out that the sequence $(A(n, M))_{n \in \mathbb{N}_{0}}$ satisfies a recurrence relation, for all $M>1$, as shown in the following result.

Theorem 2.3. For $M, n \in \mathbb{N}$, with $M>1$ or $n>1$,

$$
A(n, M)=\varphi(M) \sum_{d \mid M} A(n-1, d)\left(\frac{M}{d}\right)^{n-1} .
$$

Proof. For $n=1$, the result is a consequence of (2). When $n>1$, we can ignore the divisor 1 in the sum, since $A(n-1,1)=0$. For each divisor $d>1$ of $M$, let

$$
Y_{d}=\mathcal{A}_{n-1, d} \cap\left[1, M^{n-1} d[.\right.
$$

Also set

$$
\begin{aligned}
W & =\{c \in \mathbb{N}:(c, M)=1\} \cap[1, M[, \\
X & =\mathcal{A}_{n, M} \cap\left[1, \varphi\left(M^{n+1}\right)\right] .
\end{aligned}
$$

Observe that $\# W=\varphi(M)$ and, as $n>1$, that $Y_{1}=\emptyset$. Besides, by Proposition 2.2 . the set $Y_{d}$ has precisely $A(n-1, d)\left(\frac{M}{d}\right)^{n-1}$ elements.

Consider now the map

$$
\Phi=\left(\Phi_{1}, \Phi_{2}\right): \quad X \quad \longrightarrow\left(\bigcup_{d \mid M} Y_{d}\right) \times W
$$


defined as follows. Given $a \in X$ such that $\chi\left(\frac{a}{M}\right)=\frac{b}{d}$, where $d$ is a divisor of $M$, $b \in \mathbb{N}$ and $(b, d)=1$, take

$$
\begin{aligned}
& \Phi_{1}(a)=\frac{r}{d}, \text { where } r \text { is the remainder of the division of } b \text { by } M^{n-1} d, \\
& \Phi_{2}(a)=\text { the remainder of the division of } a \text { by } M .
\end{aligned}
$$

Notice that $\Phi_{1}(a) \in Y_{d}$ because $b \equiv r\left(\bmod M^{n-1} d\right)$, hence $b \equiv r\left(\bmod d^{n}\right)$, and, as ord $\left(\frac{b}{d}\right)=n-1$, by Proposition 2.2 we also have ord $\left(\frac{r}{d}\right)=n-1$.

To show that $\Phi$ is a bijection, let $d \neq 1$ be a divisor of $M$ and $\left(\frac{r}{d}, c\right) \in Y_{d} \times W$. Then, for $a \in X$,

$$
\begin{aligned}
\Phi(a)=\left(\frac{r}{d}, c\right) & \Longleftrightarrow\left\{\begin{array}{l}
\Phi_{1}(a)=\frac{r}{d} \\
\Phi_{2}(a)=c
\end{array}\right. \\
& \Longleftrightarrow\left\{\begin{array}{l}
d \frac{a}{M}\left\lceil\frac{a}{M}\right\rceil \equiv r \quad\left(\bmod M^{n-1} d\right) \\
\exists y \in \mathbb{N}, 1 \leq y<M^{n}: a=M y+c .
\end{array}\right.
\end{aligned}
$$

As $\chi\left(\frac{a}{M}\right)$ is an irreducible fraction with denominator $d$, then $\left\lceil\frac{a}{M}\right\rceil$ (which is equal to $y+1)$ must be a multiple of $\frac{M}{d}$, and so

$\Phi(a)=\left(\frac{r}{d}, c\right) \Longleftrightarrow\left\{\begin{array}{l}\exists y \in \mathbb{N}, 1 \leq y<M^{n}, \exists k \in \mathbb{N}: y+1=k \frac{M}{d} \wedge a=M y+c \\ \frac{M^{2}}{d} k^{2}+(c-M) k \equiv r \quad\left(\bmod M^{n-1} d\right)\end{array}\right.$

This last quadratic congruence is equivalent to a system of congruences of the form

$$
\frac{M^{2}}{d} k^{2}+(c-M) k \equiv r \quad\left(\bmod p^{t}\right),
$$

where $p$ is a prime that divides $M$ with multiplicity $t \in \mathbb{N}$. But each of these congruences has a (unique) solution (modulo $p^{t}$ ) (details in [5]). This is due to the fact that this congruence reduces modulo $p$ to $c k \equiv r(\bmod p)$, and $c \not \equiv 0$ $(\bmod p)$ since $c \in W$; moreover, the formal derivative modulo $p$ of the quadratic polynomial on $k$ is $(c-M)$, which is not a multiple of $p$. Using the Chinese Remainder Theorem, we conclude that there exists a (unique) $k \in \mathbb{N}$ such that $1 \leq k \leq M^{n-1} d$ and $\frac{M^{2}}{d} k^{2}+(c-M) k \equiv r\left(\bmod M^{n-1} d\right)$. Besides, as $(r, M)=1$, we have $k<M^{n-1} d$. Therefore, from $\frac{a}{M}<\left\lceil\frac{a}{M}\right\rceil=y+1=k \frac{M}{d}$, we deduce that $a<k \frac{M^{2}}{d}<M^{n+1}$.

As a consequence of the surjectivity of the function $\Phi$ defined in the proof just presented, we have that:

Remark 2.1. If $n \in \mathbb{N}$ and $\left(q_{j}\right)_{j \leq n}$ is a finite sequence of positive integers where $q_{j+1}$ divides $q_{j}$ for any $j$, then there exists $x \in \mathbb{Q}$ such that $\chi^{j}(x)=\frac{p_{j}}{q_{j}}$ with $\left(p_{j}, q_{j}\right)=1$.

In the particular case of $M$ being equal to a power of a prime, we get a closed formula for $A(n, M)$.

Corollary 2.4. Given a prime $p$ and $k, n \in \mathbb{N}$, then $A\left(n, p^{k}\right)=\left(\begin{array}{c}n+k-2 \\ n-1\end{array}\right)\left(\varphi\left(p^{k}\right)\right)^{n}$.

Proof. Firstly recall that the map $\varphi$ verifies

$$
\varphi\left(x^{k+1}\right)=x^{k} \varphi(x), \quad \text { for } x, k \in \mathbb{N} .
$$


As mentioned before, $A(1, M)=\varphi(M)$, and therefore the formula is valid for $n=1$ and all $k \in \mathbb{N}$. Let us proceed by induction on $n$. If $k \in \mathbb{N}$ and $n \geq 1$, then, by Theorem 2.3, we have

$$
\begin{aligned}
A\left(n+1, p^{k}\right) & =\varphi\left(p^{k}\right) \sum_{d \mid p^{k}}\left(\frac{p^{k}}{d}\right)^{n} A(n, d) \\
& =\varphi\left(p^{k}\right) \sum_{i=1}^{k} p^{(k-i) n}\left(\begin{array}{c}
n+i-2 \\
n-1
\end{array}\right) \varphi\left(p^{i}\right)^{n}, \quad \text { by induction } \\
& =\varphi\left(p^{k}\right)^{n+1} \sum_{i=1}^{k}\left(\begin{array}{c}
n+i-2 \\
n-1
\end{array}\right), \quad \text { by (4) } \\
& =\left(\begin{array}{c}
n+k-1 \\
n
\end{array}\right) \varphi\left(p^{k}\right)^{n+1} .
\end{aligned}
$$

Using the recurrence formula given by Theorem 2.3 , we have easily obtained the values of $A(n, M)$, with $1 \leq n \leq 5$ and $M \leq 20$, as displayed in Figure 2

\begin{tabular}{|l||l|l|l|l|l|l|l|l|l|l|l|}
\hline n & 2 & 3 & 4 & 5 & 6 & 7 & 8 & 9 & 10 & 11 & 12 \\
\hline \hline 1 & 1 & 2 & 2 & 4 & 2 & 6 & 4 & 6 & 4 & 10 & 4 \\
\hline 2 & 1 & 4 & 8 & 16 & 18 & 36 & 48 & 72 & 68 & 100 & 112 \\
\hline 3 & 1 & 8 & 24 & 64 & 86 & 216 & 384 & 648 & 628 & 1000 & 1424 \\
\hline 4 & 1 & 16 & 64 & 256 & 354 & 1296 & 2560 & 5184 & 5060 & 10000 & 13952 \\
\hline 5 & 1 & 32 & 160 & 1024 & 1382 & 7776 & 15360 & 38880 & 39124 & 100000 & 120768 \\
\hline
\end{tabular}

\begin{tabular}{|l||l|l|l|l|l|l|l|l|}
\hline $\mathrm{M}$ & 13 & 14 & 15 & 16 & 17 & 18 & 19 & 20 \\
\hline 1 & 12 & 6 & 8 & 8 & 16 & 6 & 18 & 8 \\
\hline 2 & 144 & 150 & 240 & 256 & 256 & 270 & 324 & 416 \\
\hline 3 & 1728 & 2058 & 3872 & 5120 & 4096 & 5670 & 5832 & 9952 \\
\hline 4 & 20736 & 24774 & 52800 & 81920 & 65536 & 93798 & 104976 & 184576 \\
\hline 5 & 248832 & 287466 & 668288 & 1146880 & 1048576 & 1396278 & 1889568 & 3048576 \\
\hline
\end{tabular}

Figure 2. The value of $A(n, M)$ for $1 \leq n \leq 5$ and $M \leq 20$.

The proof of the previous theorem provides an algorithm to explicitly compute all the congruence classes of all elements $a \in \mathbb{Z}$ such that $\frac{a}{M}$ has a certain order. This might be used to decide whether a rational number has a given order $n$, but it has the drawback that it would require solving a number of congruences that grows exponentially with $n$.

Example 2.5. From Corollary 2.4, we know that, if $p$ is prime and $n \in \mathbb{N}$, then $A(n, p)=(p-1)^{n}$. This means that the set of irreducible fractions $\frac{a}{p}$ with order $n$ is a disjoint union of $(p-1)^{n}$ arithmetic progressions of ratio $p^{n+1}$. For instance, 
with $a \in \mathbb{Z}$,

$$
\begin{aligned}
& \operatorname{ord}\left(\frac{a}{2}\right)=n \Longleftrightarrow a \equiv 2^{n}+1 \quad\left(\bmod 2^{n+1}\right) \\
& \operatorname{ord}\left(\frac{a}{3}\right)=1 \quad \Longleftrightarrow \quad a \equiv 7,8 \quad\left(\bmod 3^{2}\right) \\
& \operatorname{ord}\left(\frac{a}{3}\right)=2 \quad \Longleftrightarrow \quad a \equiv 4,11,14,19 \quad\left(\bmod 3^{3}\right) \\
& \operatorname{ord}\left(\frac{a}{3}\right)=3 \quad \Longleftrightarrow \quad a \equiv 13,22,55,56,59,64,74,77 \quad\left(\bmod 3^{4}\right) \\
& \operatorname{ord}\left(\frac{a}{3}\right)=4 \quad \Longleftrightarrow \quad x \equiv 20,23,40,83,86,109,118,128,131,157,163,172, \\
& \cos \left(\frac{a}{5}\right)=1 \quad \Longleftrightarrow \quad a \equiv 21,22,23,24 \quad\left(\bmod 5^{2}\right) \\
& \operatorname{ord}\left(\frac{a}{5}\right)=2 \quad \Longleftrightarrow \quad a \equiv 18,29,32,37,44,52,56,58,66,78,86,92,101,109, \\
& \quad 113,114 \quad\left(\bmod 5^{3}\right)
\end{aligned}
$$

\section{NuMBERS OF INFINITE ORDER}

We could not find any number outside ]0,1[ with infinite order. Nevertheless, we were able to show that almost all numbers have finite order, whose proof is the aim of this section.

Proposition 2.2 yields that, given $M \in \mathbb{N}, a \in \mathbb{Z}$ with $(a, M)=1$ and $x=\frac{a}{M}$,

a) for any $n \in \mathbb{N}_{0}$, the probability that $x$ has order $n$ is $\frac{A(n, M)}{\varphi\left(M^{n+1}\right)}$;

b) the probability that $x$ has finite order equals $\sum_{n=0}^{\infty} \frac{A(n, M)}{\varphi\left(M^{n+1}\right)}$.

Let us compute this probability.

Theorem 3.1. If $M \in \mathbb{N}$, then the probability that $\frac{a}{M}$, with $(a, M)=1$, has finite order is equal to 1.

Proof. From the meaning of $A(n, M)$ it is clear that the partial sums of the series $\mathcal{P}(M)=\sum_{n=0}^{\infty} \frac{A(n, M)}{\varphi\left(M^{n+1}\right)}$ are bounded by 1 , and so the series converges, for any given $M$. We need to show that its sum is 1 . We will prove this by induction on $M$. Obviously $\mathcal{P}(1)=1$, as $A(0,1)=1$ and $A(n, 1)=0$ if $n \geq 1$. Consider $M>1$ and 
assume that $\mathcal{P}\left(M^{\prime}\right)=1$ for all $M^{\prime}<M$. Then

$$
\begin{aligned}
\mathcal{P}(M) & =\sum_{n=1}^{\infty} \frac{A(n, M)}{\varphi\left(M^{n+1}\right)}, \quad \text { as } A(0, M)=0 \\
& =\frac{1}{M} \sum_{n=1}^{\infty} \sum_{d \mid M} \varphi(d) \frac{A(n-1, d)}{\varphi\left(d^{n}\right)}, \quad \text { by (3) and (4) } \\
& =\frac{1}{M} \sum_{d \mid M} \varphi(d) \sum_{n=1}^{\infty} \frac{A(n-1, d)}{\varphi\left(d^{n}\right)} \\
& =\frac{1}{M} \sum_{d \mid M} \varphi(d) \sum_{n=0}^{\infty} \frac{A(n, d)}{\varphi\left(d^{n+1}\right)} .
\end{aligned}
$$

By hypothesis, $\sum_{n=0}^{\infty} \frac{A(n, d)}{\varphi\left(d^{n+1}\right)}=\mathcal{P}(d)=1$ if $d<M$; therefore, using Gauss' Lemma

$$
\sum_{d \mid x} \varphi(d)=x, \quad \text { for } x \in \mathbb{N}
$$

we deduce that

$$
\begin{aligned}
\mathcal{P}(M) & =\frac{1}{M}\left(\sum_{d \mid M} \varphi(d)-\varphi(M)+\varphi(M) \mathcal{P}(M)\right) \\
& =\frac{1}{M}(M-\varphi(M)+\varphi(M) \mathcal{P}(M)) \\
& =1-\frac{\varphi(M)}{M}+\frac{\varphi(M)}{M} \mathcal{P}(M)
\end{aligned}
$$

and so $\mathcal{P}(M)=1$, as $\varphi(M)<M$.

Remark 3.1. From this theorem it is easy to infer that, for any denominator $M$ and $n \in \mathbb{N}$,

(1) $\lim _{k \rightarrow+\infty} \frac{\#\left\{1 \leq a \leq k: a \in \mathcal{A}_{n, M}\right\}}{k}=\frac{A(n, M)}{M^{n+1}}$.

(2) For any $M \in \mathbb{N}, \lim _{k \rightarrow+\infty} \frac{\#\left\{1 \leq a \leq k: \exists n \in \mathbb{N}_{0}: a \in \mathcal{A}_{n, M}\right\}}{k}=\frac{\varphi(M)}{M}$.

(3) The density of the integers whose orbits does not reach $\mathbb{Z}$ is zero.

Corollary 3.2. There is no infinite arithmetic progression in $\mathbb{Q}$ whose elements have infinite order.

Proof. Any arithmetic progression in $\mathbb{Q}$ contains an arithmetic progression of the form $\left(\frac{s+n r M}{M}\right)_{n \in \mathbb{N}}$, with $M \in \mathbb{N}, s, r \in \mathbb{Z}$ and $(s, M)=1$. In that case, we note that $(s+n r M, M)=1$ and that a number of the form $\frac{a}{M}$, with $(a, M)=1$, has probability $\frac{1}{2 r M}$ of belonging to this arithmetic progression. Using Theorem 3.1 we conclude that the arithmetic progression must include elements of finite order. 
For a while we were tempted to believe that if, for a fixed $M \in \mathbb{N}$ and for all $n \in$ $\mathbb{N}_{0}$, one has $c_{n}$ disjoint congruence classes modulo $M^{n+1}$ such that $\sum_{n \geq 0} \frac{c_{n}}{M^{n+1}}=1$, then the union of all those classes is all $\mathbb{Z}$, with the possible exception of a finite set. This, however, is not true, as can be confirmed by the next example, in which we chose $M=3$ just to simplify matters, but where we could just as well have taken an arbitrary $M$.

Example 3.3. The idea is to show that one can inductively construct, for each $n \in \mathbb{N}_{0}$ and $k \in\left\{1,2,3, \ldots, 2^{n}\right\}$, an element $x_{n, k}$ in $\left\{1,2, \ldots, 3^{n+1}\right\}$ in such a way that, if $(n, k) \neq(m, s)$, then the classes modulo $3^{\min \{m, n\}+1}$ of $x_{n, k}$ and $x_{m, s}$ are disjoint. Moreover, one wants to choose those elements so that the union of all these congruence classes does not contain any number of the set $Y=\left\{1+3^{n}: n \in \mathbb{N}_{0}\right\}$, for example.

We start with $x_{0,1}=3$. Now, for a given $n \geq 1$, suppose that we have already defined $x_{m, k} \in\left\{1,2, \ldots, 3^{m+1}\right\}$, for all $m<n$, satisfying the above mentioned conditions. In the set $\left\{1,2 \ldots, 3^{n+1}\right\}$, there are $n+1$ elements of $Y$ and, for each $0 \leq i \leq n-1$, we have $3^{n-i}$ elements in the class of $x_{i, j}$ modulo $3^{i+1}$. We thus have a total of $(n+1)+3^{n}+2 \times 3^{n-1}+2^{2} \times 3^{n-2}+\cdots+2^{n-1} \times 3$, that is $3^{n+1}-3 \times 2^{n}+(n+1)$ elements already "used". Then $x_{n, k}$, for $1 \leq k \leq 2^{n}$, can be selected among the remaining $3^{n+1}-\left(3^{n+1}-3 \times 2^{n}+(n+1)\right)$ elements of $\left\{1,2 \ldots, 3^{n+1}\right\}$. This is possible since $3^{n+1}-\left(3^{n+1}-3 \times 2^{n}+(n+1)\right)=3 \times 2^{n}-(n+1) \geq 2^{n}$.

We therefore obtain $2^{n}$ classes modulo $3^{n+1}$, for all $n \in \mathbb{N}_{0}$, which are all disjoint and whose complement contains the infinite set $Y$.

This example shows that if indeed it is true that all rational numbers bigger that 1 have finite order, as the numerical computations suggest, then in order to prove it one has to better understand the relationships among the congruence classes that make up the sets $\mathcal{A}_{n, M}$.

\section{An efFicient ALGORITHM}

In this section we describe a simple algorithm that verifies if a rational number has an order less than a fixed bound. It was precisely this algorithm that allowed us to obtain the results presented in the previous tables. It runs very quikly, as long as the computer is able to store the appropriate numbers.

The strategy behind this procedure is the following. Take $\frac{a}{M}$ and consider the sequence $\left(a_{n}\right)_{n \in \mathbb{N}_{0}}$ defined by

$$
\begin{aligned}
a_{0} & =a \\
a_{n+1} & =M \chi\left(\frac{a_{n}}{M}\right) .
\end{aligned}
$$

If we know that $\frac{a}{M}$ has order less or equal to $N$, then, for $1 \leq s<N$, the order of $\frac{a_{s}}{M}$ is less or equal to $N-s$. Hence, using Proposition 2.2. we can replace $a_{s}$ by the remainder of the division of $a_{s}$ by $M^{N+1-s}$. The order of $\frac{a}{M}$ will then be the first $s$ such that $a_{s}$ is a multiple of $M$. We summarize this as follows:

Algorithm: Given $M \in \mathbb{N}, a \in \mathbb{Z}$ and $N \in \mathbb{N}$, consider $\frac{a}{M}$ and define the sequence $\left(r_{n}\right)_{n \in \mathbb{N}_{0}}$ as

$$
\begin{aligned}
r_{0} & =\text { the remainder of the division of } a \text { by } M^{N+1} \\
r_{n+1} & =\text { the remainder of the division of } M \chi\left(\frac{a_{n}}{M}\right) \text { by } M^{N+1-s} .
\end{aligned}
$$


Then

$$
\operatorname{ord}\left(\frac{a}{M}\right)= \begin{cases}k, & \text { if } \exists k \leq N: M \mid r_{k} \text { and } M \nmid r_{s}, \text { for all } s<k, \\ >N, & \text { otherwise. }\end{cases}
$$

We highlight the fact that this algorithm only needs to deal with numbers of length less or equal to $M^{N+1}$, and that each step reduces the bounds involved.

\section{OTHER APPROACHES}

An alternative approach to study the dynamics of the map $\chi$ would be to use the finite expansions of the successive numerators in the bases given by the respective denominators. One of the problems with this procedure is that $\chi$ involves a multiplication in which carries intervene. In the particular case where the initial point is a fraction with denominator equal to a prime number $p$, and without the carries, the dynamics of $\chi$ would be one of an infinite dimensional linear cocycle with base space equal to the space of almost zero sequences on $p$ symbols and fibers over the field $\mathbb{F}_{p}$. This sounds already intricate, but the interference of the carries is a source of additional difficulties, causing $\chi$ to resemble a generalized shift with sensitive dependence on initial conditions [2]. This seems to hint, once more, that the question of determining whether or not there are rational numbers bigger than 1 of infinite order may be a hard problem.

For each prime $p$ there is a map related to $\chi$ that can be defined on the field of $p$-adic numbers as follows. Given

$$
x=\sum_{j \geq k} a_{j} p^{j} \in \mathbb{Q}_{p}
$$

where $k \in \mathbb{Z}$ and $a_{j} \in\{0,1, \ldots, p-1\}$ for all $j$, put

$$
\lceil x\rceil_{p}=1+\sum_{j \geq 0} a_{j} p^{j} .
$$

Now let $\chi_{p}: \mathbb{Q}_{p} \rightarrow \mathbb{Q}_{p}$ be the map defined by $\chi_{p}(x)=x\lceil x\rceil_{p}$, which clearly coincides with the quasi-quadratic map when restricted to the rational numbers whose denominator is a power of $p$ and that are not integers. Note that this map does not agree with $\chi$ on the integers, but has the advantage of being continuous.

One can now consider the question of knowing if, for any $p$-adic number $x$, there exists $n \in \mathbb{N}_{0}$ such that $\chi_{p}^{n}(x)$ is a $p$-adic integer. It turns out that, in this case, there are elements of infinite order other than the rational numbers in ]0,1[. This can be seen as follows. By an argument similar to the one used to prove Theorem 2.3. we may verify that, given $k, n \in \mathbb{N}$, the set

$$
\Lambda_{k, n}=\left\{a \in \mathbb{Z}_{p}: \chi_{p}^{n}\left(\frac{a}{p^{k}}\right) \notin \frac{1}{p^{k-1}} \mathbb{Z}_{p}\right\}
$$

is nonempty and a finite union of congruence classes in $\mathbb{Z}_{p}$ modulo $p^{(n+1) k}$, and so compact as well. It is clear that $\Lambda_{k, n+1} \subset \Lambda_{k, n}$. Moreover, one can show that the disjoint congruence classes that constitute $\Lambda_{k, n+1}$ are equally distributed inside the ones that form $\Lambda_{k, n}$. Therefore, for any fixed $k$, the set $\bigcap_{n \in \mathbb{N}} \Lambda_{k, n}$ is nonempty, and since it is the intersection of a nested sequence of compact nonempty sets, each one formed by an increasing number of balls with decreasing radius, that are scattered through the balls of the previous set, we see that this intersection is a perfect subset 
(with zero Haar measure) of the locally compact group $\mathbb{Q}_{p}$. So, it is uncountable, and hence there are elements of infinite order in $\mathbb{Q}_{p}$ besides the ones in $\left.\mathbb{Q} \cap\right] 0,1[$.

Moreover, as $\chi_{3}$ is the polynomial $x^{2}+x$ in $\mathbb{Z}_{3}$, and it is continuous, the wild nature of the orders we found among the fractions $\frac{a}{3}$ (see Fig. 11) may somehow be related to the chaotic behaviour of $x^{2}+x$ in $\mathbb{Z}_{3}$, as described in 1 .

\section{Final comments and Remarks}

Given a fixed integer $M$, suppose one randomly chooses an integer $a_{1}$, sets $M_{1}=$ $M /\left(M, a_{1}\right)$, and then repeats the process by randomly choosing $a_{2}$, leeting $M_{2}=$ $M_{1} /\left(M_{1}, a_{2}\right)$, and so on. Note that this process somehow generalizes what happens to the successive denominators of $\chi\left(\frac{a}{M}\right)$. Given $M$ and $n$, consider now the question of determining the probability that $M_{n}=1$. Denote this probability by $\mathcal{P}(n, M)$. Clearly: $\mathcal{P}(1, M)=1 / M ; \mathcal{P}(0, M)=1$ if $M=1$, and $\mathcal{P}(0, M)=0$ otherwise; if $p$ is a prime number, then $\mathcal{P}(n, p)=(1-1 / p)^{n-1} / p$. It is easy to show that the numbers $P(n, M)$ satisfy the following recurrence relation

$$
\mathcal{P}(n, M)=\sum_{d \mid M} \frac{\varphi(d)}{M} \mathcal{P}(n-1, d),
$$

that is also satisfied by the numbers $A(n, M) / \varphi\left(M^{n+1}\right)$, as can easily be shown by induction on $M$ using Theorem 1. Thus, the probability that a rational number $\frac{a}{M}>1$, with $(a, M)=1$, has order $n$ under $\chi$ is exactly the same that the random process just described ends up after $n$ steps, when starting with $M$. This reveals that the map $\chi$ behaves, at least in this respect, just like a random procedure.

There are also some curious analogies between our query, if $\chi$ has no elements of infinite order, and both the Collatz problem and the Erdös-Straus conjecture. The similarities may be only superficial, but are nevertheless of some interest. In the Collatz problem, Riho Terras has shown that the set of elements that have a finite stopping time $n$, a notion analogous to our concept of order, is a disjoint union of congruence classes (see Theorem 1.2 in 4]). The issue is then whether these cover all integers. This is an open question, but Terras has also proved that the density of the integers that do not have finite stopping time is zero. Both these results are similar to what was shown in the present paper. As for the ErdösStraus conjecture, William Webb has shown in [6] that the density of the numbers for which the conjecture is false is zero, using the fact that one can prove its validity for an infinite set of congruence classes (see Lemma 2 in [6]). The connection with our problem is here less obvious, but in all three cases, ours and these other two, one could solve the respective conundrum by showing that a certain system of congruences is a covering of the integer 1 .

Finally, we mention that our methods also apply to the map $x \mapsto x\lfloor x\rfloor$, since $x\lfloor x\rfloor=\chi(-x)$, for any $x \in \mathbb{Q} \backslash \mathbb{Z}$.

\footnotetext{
${ }^{1}$ The question of whether one can prove the Erdös-Straus conjecture by showing its validity on an infinite covering system of congruences is unclear, although there are good reasons to believe it to be an approach riddled with difficulties: see Terrence Tao considerations on this matter in his blog, at http://terrytao.wordpress.com/2011/07/07/on-the-number-of-solutions-to-4p-1n_1-1n_2-1n_3
} 


\section{REFERENCES}

[1] A. H. Fan and L. M. Liao, On minimal decomposition of p-adic polynomial dynamical systems, Advances in Mathematics, Vol. 228, 4 (2011) 2116-2144.

[2] C. Moore, Generalized shifts: unpredictability and undecidability in dynamical systems, Nonlinearity 4 (1991) 199-230.

[3] G. Tenenbaum, Introduction to Analytic and Probabilistic Number Theory, Cambridge University Press, 1995.

[4] R. Terras, A Stopping Time Problem on the Positive Integers, Acta Arithmetica XXX (1976) $241-252$.

[5] I. M. Vinogradov, Elements of Number Theory, Dover 1954.

[6] W. A. Webb, On $4 / n=1 / x+1 / y+1 / z$, Proceedings of the American Mathematical Society 25 (1970) 578-584.

Department of Mathematics and Applications, University of Minho, Campus de GualTAR, 4710-057 Braga, Portugal

E-mail address: assis@math.uminho.pt

Department of Mathematics, University of Porto Rua do Campo Alegre 687, 4169-007 Porto, Portugal

E-mail address: mpcarval@fc.up.pt

Department of Mathematics, University of Porto Rua do Campo Alegre 687, 4169-007 Porto, Portugal

E-mail address: ajmachia@fc.up.pt 